\title{
Social Media Use and Mental Health During the COVID-19 Pandemic: A Meta-Analysis of 14 Cross- Sectional Studies
}

\author{
Youngrong Lee \\ Yonsei University \\ Ye Jin Jeon \\ Yonsei University \\ Sunghyuk Kang \\ Yonsei University \\ Jae II Shin \\ Yonsei University \\ Young-Chul Jung \\ Yonsei University \\ Sun Jae Jung ( $\sim$ SUNJAEJUNG@yuhs.ac ) \\ Yonsei University
}

\section{Research Article}

Keywords: Anxiety, Depression, Social Distance, Mental Health, Systemic Reviews

Posted Date: February 14th, 2022

DOI: https://doi.org/10.21203/rs.3.rs-1270456/v1

License: () (1) This work is licensed under a Creative Commons Attribution 4.0 International License.

Read Full License 


\section{Abstract}

Background: Public isolated due to the early quarantine regarding coronavirus disease 2019 (COVID-19) increasingly used more social media platforms. Contradictory claims regarding the effect of social media use on mental health needs to be resolved. We aimed to summarise the association between the time spent on social media platform during the COVID-19 quarantine and mental health outcomes (i.e., anxiety and depression).

Methods: We searched 327 studies from the PubMed, Embase, and Cochrane Library databases. These studies evaluated the association between use of social media platform and mental health outcomes, including anxiety and depression, ascertained by various screening tools. We summarised the final 14 studies in terms of the odds ratios (ORs). Data analyses included a random-effect model and an assessment of inter-study heterogeneity. This meta-analysis review was registered in PROSPERO (https://www.crd.york.ac.uk/PROSPERO/, registration No CRD42021260223, 15 June 2021).

Results: In studies regarding anxiety, overall studies had a pooled OR of 1.55 (95\% Cl: $1.30-1.85$; OR that would be categorized as having anxiety when increasing from "less" to "frequent") and mild inter-study heterogeneity $(12=26.77 \%)$. In studies regarding depression, overall studies had a pooled OR of 1.43 (95\% Cl: 1.14-1.80; OR that would be categorized as having depression when increasing from "less" to "frequent") and moderate heterogeneity $(12=67.16 \%)$.

Conclusions: Our analysis demonstrated that the excessive time spent on social media platform was associated with a greater likelihood of having symptoms of anxiety and depression.

\section{Introduction}

In the first half-year of the virus pandemic, more than 100 million people were confirmed with coronavirus disease 2019 (COVID-19), and approximately 500 thousand have died [1]. Despite the widespread vaccination worldwide, the coronavirus outbreak is not expected to dampen due to the continuous emergence of new viral strains and difficulty in effective quarantine interventions. As a result of strong quarantine measures, private meetings, gatherings, and physical contact with intimate relatives have been reduced [2]. Prolonged social distancing and loss of intimate interpersonal contact increase feelings of frustration, boredom, anxiety, and potentially depression [3]. Social media is reportedly considered as a channel of communication that could relieve negative aspects of isolation through helping people escape negative emotions [4], projecting their personality as they desire, and evoking the impression of gaining back some control [5].

Social media may be helpful for relieving anxiety and depression by providing information regarding the pandemic [6, 7]. However, prolonged use of social media by the isolated could be a double-edged sword that can adversely affect mental health due to sustained exposure to excessive information and misinformation [8-10]. Due to conflicting evidence and views regarding the effect of social media 
platform on the mental health, the recommendation for the use of social media in the era of COVID-19 has been questioned.

Therefore, we conducted a meta-analysis to confirm the contradictory effects of social media platform on anxiety and depression based on studies reporting an association between the use of social media and mental health outcomes (i.e., anxiety and depression) on the pandemic setting.

\section{Methods}

\section{Study selection}

A systematic literature search of the PubMed, Embase, and Cochrane Library databases was performed to identify studies published before December 20,2020. We conducted a systematic search with the following search terms with no language restrictions: (1) ("COVID-19" OR "corona") AND ("mental health" OR depress* OR anxiety) AND ("social media" OR "Instagram" OR "Facebook" OR "twitter") for PubMed, (2) ("coronavirus disease 2019'/exp/mj) AND ("mental health"/exp/mj OR "depression"/exp OR "anxiety"/exp) AND ("social media"/exp/mj OR "Facebook"/exp OR "twitter"/exp OR "Instagram"/exp) for Embase; (3) ("COVID-19" OR "corona")AND("mental health" OR depress* OR "anxiety") AND ("social media" OR

'Instagram" OR "Facebook" OR "twitter") for Cochrane Library. Articles were first screened by reviewing titles, followed by a full-text review. Every selection stage involved three independent researchers (two medical doctors [SJJ and YRL] and one graduate student from the Epidemiology Department [YJJ]). Every article was independently evaluated by two researchers (YJJ and YRL), and a third researcher (SJJ) mediated the final selection in case of differences in opinion. Study data were extracted by two independent researchers (YRL and YJJ). Studies were subdivided into categories according to the summary estimate of effect sizes (odds ratio [OR], beta estimate from multiple linear regression [ $\beta$ ], and correlation coefficient [Pearson's r]).

\section{Inclusion and exclusion criteria}

Studies included data regarding the time spent on social media during the COVID-19 pandemic and various mental health symptoms (e.g., anxiety, depression, psychological distress, fear, panic, posttraumatic stress symptoms, rumination, vicarious traumatisation, life satisfaction, information avoidance, sense of inadequacy, and perceived societal risk).

Included studies regarding depressive symptoms were measured with diagnostic tools such as the Patient Health Questionnaire-9 (PHQ-9), shortened version of PHQ (PHQ-2) [11], General Health Questionnaire-28 (GHQ-28) [12], Depression Anxiety and Stress Scales (DASS-21) [13], or the Chinese version of WHO-Five Well-Being Index (WHO-5) [14]. Studies regarding anxiety symptoms were measured with diagnostic tools such as the Generalized Anxiety Disorder-7 (GAD-7), shortened version of GAD (GAD-2) [15], or GHQ-28. 
The initial literature search was intended to include all types of observational study designs (cohort, casecontrol, and cross-sectional studies). The included studies were all cross-sectional studies. Case reports, letters, comments, and narrative reviews without quantitative results were not included. Studies were excluded when they examined traditional social media (television and radio). Studies without clear explanations of variable measurements were excluded. We included studies that investigated the association between time spent on social media and mental health outcomes (e.g., anxiety and depression) (see also Supplementary Material 1). While final included studies presented their results in either OR, $\beta$, and/or Pearson's $r$ (Supplementary material 1), the pooled effect size of this meta-analysis was mainly presented in an odds ratio.

\section{Exposure variables}

The final 14 studies measured the amount of time spent on social media, which was either categorical or continuous variables (see Supplementary Material 1). It was measured based on the response to an item in the questionnaire: "How often were you exposed to social media? [categorical]" and "How long (in hours) were you exposed to social media? [continuous]" The exposure was measured in different wordings as follows: "Less" vs. "Frequently," "Less" vs. "Often", "less than 1 hour" vs. "2 hours or more," or "less than 3 hours" vs. " 3 hours or more." To calculate the overall effect, we operationally redefine these individually measured exposure levels (e.g., "Less" and "Few" were considered the same as "less than 2 hours;" "less than 1 hour," "Frequently," and "Often" were treated the same as " 2 hours or more" and "3 hours or more").

\section{Outcome variables}

The outcomes of included studies were "anxiety", and "depression". Anxiety was ascertained by using GAD-7 (cut-off: 10+), DASS-21, and PHQ-9, while depression was measured using PHQ-9 (cut-off: 10+), WHO-5 (cut-off: 13+), and GHQ-28 (cut-off: 24+). Anxiety and depression measured by using screening tools with cut-offs presented results in odds ratios (see Supplementary Material 1).

\section{Statistical analysis}

Each included study reported summary statistics in different ways. Six studies that dealt with anxiety symptoms and seven with depression (Supplementary Material 1-1-1, 1-2-1) reported ORs and their $95 \%$ confidence intervals (Cls) ( $n=9,579$ and $n=13,241$ for anxiety and depressive symptoms, respectively). Three studies each on anxiety and depression (Supplementary Material 1-1-2, 1-2-2) reported their findings in $\beta$ ( $n=2,376$ and $n=2,574$ for anxiety and depression, respectively). The remaining studies reported Pearson's $r$ ( $n=2,483$ for anxiety). We presented our pooled effect sizes of the meta-analysis in odds ratio and its $95 \%$ Cls (Figure 2), and other pooled statistics such as $\beta$ and Pearson's r were presented in Supplementary material 2.

We used each estimate in the final adjusted model. All statistical analyses and visualisations were performed with the "meta," "metaphor," and "dmeter" package of R version 3.6.3 (https://cran.r- 
project.org/), using a random-effect model [16-18]. We calculated the pooled effect sizes, Cochrane's Q, and $\mathrm{I}^{2}$ to assess heterogeneity. The pooled effect sizes, Cls, and prediction intervals were calculated by estimating the pooled effect and Cls using the Hartung-Knapp-Sidik-Jonkman method, which is known as the one of the most conservative methods [19]. The degree of heterogeneity was categorised as low, moderate, or high with threshold values of $25 \%, 50 \%$, and $75 \%$, respectively [20]. Possible causes of heterogeneity among study results were explored by statistical methods such as influential analysis, the Baujat plot, leave-one-out analysis, and Graphic Display of Heterogeneity analysis [21]. In addition, publication bias was assessed using funnel plots, Egger's tests, and the trim-and-fill method [22].

\section{Quality assessment}

Quality assessment was conducted by two independent researchers, a psychiatrist (SHK) and an epidemiologist (YRL), using the Risk of Bias Assessment Tool for Nonrandomized Studies (RoBANS), which can assess cross-sectional studies [23]. RoBANS has been validated with moderate reliability and good validity. RoBANS applies to cross-sectional studies and comprises six items: participant selection, confounding, exposure measurement, blinding of outcome assessments, missing outcomes, and selective reporting of outcomes. Each item is measured as having a "high risk of bias," "low risk of bias," or "uncertain." For example, based on "participant selection," each researcher marked an article as having a "high risk of bias" if, for example, the patient definitions of depression were generated by self-reported data. In cross-sectional studies, misclassification cases due to an unreliable self-contained questionnaire for categorizing depressive patients were rated as "high risk." For the qualitative assessment, we classified a study as "low quality" if there were two or more "high risk of bias" grades. The study was rated as "high quality" only if the evaluation of both raters was congruent. For sensitivity analysis, we repeated our analysis including only "high quality" studies and compared it with the pooled estimates of overall results.

\section{Ethical approval}

No ethical approval and patient consent are required since this study data is based on published literature. This meta-analysis review was registered with PROSPERO (https://www.crd.york.ac.uk/PROSPERO/, registration No CRD42021260223, 15 June 2021).

\section{Results}

\section{Included and excluded studies}

We selected 346 studies from the database search (288 from PubMed, 34 from Embase, and 24 from the Cochrane Library). After removing 19 duplicate publications, 327 studies were included for the title and full-text review. Non-original studies and those conducted with irrelevant subjects $(n=218)$ were excluded. Another 95 studies were excluded finally due to inconsistent study estimates. Each of the final 14 studies measured multiple mental health outcome variables (i.e., anxiety and depression), and pooled effect sized were calculated for each outcome variable. Figure 1 shows the number of included 
publications for each mental health outcome. All included studies were cross-sectional studies. The pooled effect size was presented in odds ratio.

Of studies ascertaining anxiety, six studies used the GAD, three used the DASS-21, and the rest were measured by PHQ-9 and GHQ-28. Of the 9 papers regarding depression, five studies used PHQ-9 (cut-off: $10+)$, three used DASS-21, and the rest used Chinese version of WHO-5. Of the seven papers on psychological distress, DASS-21 was used in four papers for measuring stress (see also Supplementary Material 1 for more details).

\section{Time spent on social media and mental health outcomes}

Table 1 shows the result of the meta-analysis about the relationship between time spent on social media and mental health outcomes (i.e., anxiety and depression) of the selected cross-sectional studies. In anxiety studies, overall studies had a pooled odds ratio of 1.55 (95\% Cl: $1.30-1.85$; OR that would be categorized as having anxiety when increasing from "less" to "frequent") and mild inter-study heterogeneity $\left(I^{2}=26.77 \%\right)$ (Fig. 2). The three cross-sectional studies (presented in $\beta$ ) were insignificant ( $\beta=0.05,95 \% \mathrm{Cl}:-0.32-0.15$; a unit increment of each screening tool score per hour) with relatively high inter-study heterogeneity $\left(I^{2}=76.07 \%\right)$. The overall estimate of the four cross-sectional studies (Pearson's r) was 0.18 (95\% Cl: 0.10-0.27) with high inter-study heterogeneity $\left(I^{2}=73.04 \%\right)$. In studies about depression, overall studies had a pooled Odds ratio of 1.43 (95\% Cl: 1.14-1.80; Odds ratio that would be categorized as having depression when increasing from "less" to "frequent") and moderate heterogeneity $\left(I^{2}=67.16 \%\right)$ (Fig. 2). 
Table 1. Association between social media use and anxiety ${ }^{\mathrm{a}}$ and depression ${ }^{\mathrm{b}}$

Studies, $\mathbf{n} \quad$ Pooled

(participants) effect sizes $[95 \% \mathrm{Cl}] \quad$ Q-statistics $\quad \mathrm{I}^{2}(\%)$

\section{Anxiety symptoms}

Odds ratio

Overall studies

6 (9579)

$1.55[1.30-1.85]$

6.84

26.94

Quality assessment

\begin{tabular}{lllll} 
High Quality & $\mathbf{4 ( 7 5 9 9 )}$ & $\mathbf{1 . 4 5}[\mathbf{1 . 2 1 - 1 . 7 3}]$ & 1.76 & 0.00 \\
\hline$\beta^{c}$ & $3(2376)$ & $0.05[-0.32-0.41]$ & 8.36 & 76.07 \\
\hline Pearson's r ${ }^{\text {d }}$ & $\mathbf{4 ( 2 4 8 3 )}$ & $\mathbf{0 . 1 8}[\mathbf{0 . 1 0 - 0 . 2 7 ]}$ & 11.13 & 73.04
\end{tabular}

\section{Depressive symptoms}

Odds ratio

Overall studies

$6(13241)$

$1.43[1.14-1.80]$

15.22

67.16

Quality assessment

\begin{tabular}{|c|c|c|c|c|}
\hline High Quality & $2(4481)$ & $1.42[0.69-2.90]$ & 0.33 & 0.00 \\
\hline$\beta^{c}$ & $3(2574)$ & $0.08[0.01-0.14]$ & 0.82 & 0.00 \\
\hline
\end{tabular}

a Anxiety symptoms were ascertained using the GAD-7 (cut-off: 10), GHQ-28, GAD-2, PHQ-4, GAD-2, SAS, and DASS-21.

${ }^{b}$ Depressive symptoms were ascertained by the DASS-21, WHO-5 (cut-off: 13), PHQ-9 (cut-off:10), GHQ-28, and PHQ-2.

${ }^{\mathrm{c}}$ Beta value calculated by linear regression analysis indicates the change in score over time in hours.

${ }^{\mathrm{d}}$ Correlation analysis

B, Beta value; Cl, Confidence Interval; DASS, Subscale scores of Depression, Anxiety, and Stress Scale;

GAD, Generalized Anxiety Disorder; GHQ-28, General Health Questionnaire-28; PHQ, Patient Health

Questionnaire; SAS- Self-Rating Anxiety Scale

* Significant results are in bold text. 


\section{Quality assessment}

As result of quality assessment analysis, pooled effect size of studies classified as "high quality" was presented in Table 1. The results were similar to the overall outcome (anxiety: OR $=1.45,95 \%$ Cl: $1.21-$ 1.96; depression: $\mathrm{OR}=1.42,95 \% \mathrm{Cl}: 0.69-2.90)$. High-quality studies had low inter-study heterogeneity (anxiety: $I^{2}=0.00 \%$; depression: $I^{2}=0.00 \%$ ).

\section{Publication bias}

Publication bias was assessed by funnel plot analysis and Egger's test (Supplementary Material 4-1). Funnel-plot analyses revealed symmetrical results (Supplementary Material 4-2). In addition, all results of the Egger test were statistically insignificant, indicating improbable publication bias. After applying the trim-and-fill method, the funnel plot revealed no asymmetry (Supplementary Material 5), indicating no significant publication bias.

\section{Discussion}

Our study aimed to present a comprehensive direction of relevance by analysing studies investigating the association between time spent on social media during the COVID-19 pandemic and symptoms of mental health indices (e.g., anxiety, depressive, and psychological distress) among the public. By summarising the different estimates (ORs, $\beta$, and Pearson's $r$ ) presented in the cross-sectional studies, we found that time spent on social media was associated with an increased likelihood of symptoms of anxiety and depression. However, no significant association was seen with psychological distress.

Our results are consistent with previous systematic reviews and meta-analysis performed before the COVID-19 pandemic. A systematic literature review before the COVID-19 outbreak (2019) found that the time spent by adolescents on social media was associated with depression, anxiety, and psychological distress [24]. A meta-analysis of 11 studies (2017) also reported a weak association between social media use and depressive symptoms in children [25]. A meta-analysis of 23 studies (2018) reported significant correlation between social media use and psychological distress [26]. Likewise, our study also observed a similar trend of a negative effect of social media on mental health outcomes in the COVID-19 pandemic. However, the estimates of inter-study heterogeneity of these meta-analysis were relatively high (meta-analysis of 11 studies: $\mathrm{I}^{2}=92.4 \%$; meta-analysis of 23 studies: $\mathrm{I}^{2}=62.00 \%$ for anxiety, $\mathrm{I}^{2}=80.58 \%$ for depression) compared to our analysis, which implies relative homogeneity of our study population and reliable results.

Despite the benefits of social media, unverified information is freely disseminated on social media, while opinions are perceived as facts. There has been a stream of news regarding the pandemic, creating a sense of urgency and anxiety. Repeated exposure to this stream may affect the construct of external 
reality and may lead to a delusion-like experience, which has been linked to anxiety and social media overuse $[27,28]$.

Additionally, discrimination and stigma related to COVID-19 on social media can make people fearful of infection and exacerbate depression and anxiety [29]. Fear of COVID-19 may be compounded by coexisting depression and anxiety disorders [30]. Due to the high accessibility of social media and the ease of socialisation in a controlled setting, individuals with underlying depression may be more drawn to social media interactions rather than face-to-face ones, more so in the pandemic era [31].

In interpreting the findings of this study, several limitations should be considered. First, all the studies included were cross-sectional design. The possibility of a reverse causal relationship cannot be ruled out. The COVID-19 pandemic has been around for less than a year and longitudinal studies might not be under the publication process at the time of study conducted. This may explain to some extent why all included studies were cross-sectional studies. Further studies with longitudinal data are warranted. Second, our results do not represent the general population since most of the studies recruited participants through a web-based survey, which may have had a selection bias. Lastly, some of the analysis showed a relatively high inter-study heterogeneity (range: $I^{2}=0.00 \%-80.53 \%$ ). To identify the cause of heterogeneity, we summarised the results of the statistical approaches (influential analysis, Baujat plot, leave-one-out analysis, and GOSH analysis [21]) in Supplementary Material 6 and 7.

Despite these limitations, our study exhibits a number of strengths; as far as we understand, our study is the first meta-analysis to examine the relationship between social media use and mental health outcomes during the COVID-19 pandemic, to validate the results by various verification methods such as trim-and-fill methods, influential analysis, and heterogeneity analysis. In addition, various sensitivity analyses such as quality assessment were also attempted.

Our analysis demonstrates that excessive time spent on social media is associated with increased anxiety and depressive symptoms in the era of COVID-19. While social media may be considered as an alternative for alleviating some stress caused by the pandemic, our findings suggest that excessive use of social media can be detrimental for mental health. A longitudinal study to determine the true effect of social media is required.

\section{Abbreviations}

COVID-19, coronavirus disease 2019; OR, odds ratio; Cl, confidence interval; PHQ-9, Patient Health Questionnaire-9; PHQ-2, shortened version of PHQ; GHQ-28, General Health Questionnaire-28; DASS-21, Depression Anxiety and Stress Scales; WHO-5, World Health Organization-Five Well-Being Index; GAD-7, Generalized Anxiety Disorder-7; GAD-2, shortened version of GAD; RoBANS, Risk of Bias Assessment Tool for Nonrandomized Studies

\section{Declarations}


Ethical approval and consent to participate

No ethical approval and patient consent are required since this study data is based on published literature. This meta-analysis review was registered with

PROSPERO (https://www.crd.york.ac.uk/PROSPERO/, registration No CRD42021260223, 15 June 2021).

\section{Consent for publication}

Not applicable

\section{Data availability}

The data that support the findings of this study are available from the corresponding author upon reasonable request.

\section{Competing interests}

Not applicable

\section{Funding}

This study was supported by the National Research Foundation of Korea, funded by the Ministry of Science and ICT (2020R1C1C1003502), awarded to SJJ.

\section{Acknowledgements}

The authors would like to thank the Editage (www.editage.co.kr) for English language editing.

\section{Author contribution}

Conceptualization: YRL, SJJ. Data curation: SJJ, JIS, YCJ, YRL. Formal analysis: YRL, SJJ. Funding acquisition: SJJ. Methodology: JIS, YCJ, YRL, SJJ. Project administration: SJJ. Visualization: YRL. Writing - original draft: YRL, YJJ, SHK, SJJ. Writing - review \& editing: YRL, YJJ, SHK, JIS, YCJ, SJJ. The corresponding author attests that all listed authors meet authorship criteria and that no others meeting the criteria have been omitted.

\section{References}

1. Dong E, Du H, Gardner L. An interactive web-based dashboard to track COVID-19 in real time. The Lancet infectious diseases. 2020;20(5):533-4.

2. Clemens V, Deschamps P, Fegert JM, Anagnostopoulos D, Bailey S, Doyle M, et al. Potential effects of "social" distancing measures and school lockdown on child and adolescent mental health. Springer; 2020. 
3. Brooks SK, Webster RK, Smith LE, Woodland L, Wessely S, Greenberg N, et al. The psychological impact of quarantine and how to reduce it: rapid review of the evidence. The lancet. 2020;395(10227):912-20.

4. Marino C, Gini G, Vieno A. i Spada, M.(2018). A comprehensive meta-analysis on Problematic Facebook Use. Computers in Human Behavior.83(1):262-77.

5. Ryan T, Chester A, Reece J, Xenos S. The uses and abuses of Facebook: A review of Facebook addiction. Journal of behavioral addictions. 2014;3(3):133-48.

6. Liu S, Yang L, Zhang C, Xiang Y, Liu Z, Hu S, et al. Online mental health services in China during the COVID-19 outbreak. Lancet Psychiatry. 2020; 7 (4): e17-e18.

7. Liu BF, Kim S. How organizations framed the 2009 H1N1 pandemic via social and traditional media: Implications for US health communicators. Public Relations Review. 2011;37(3):233-44.

8. Fung IC-H, Tse ZTH, Cheung C-N, Miu AS, Fu K-W. Ebola and the social media. 2014.

9. Depoux A, Martin S, Karafillakis E, Preet R, Wilder-Smith A, Larson H. The pandemic of social media panic travels faster than the COVID-19 outbreak. Oxford University Press; 2020.

10. Kramer AD, Guillory JE, Hancock JT. Experimental evidence of massive-scale emotional contagion through social networks. Proceedings of the National Academy of Sciences. 2014;111(24):8788-90.

11. Richardson LP, McCauley E, Grossman DC, McCarty CA, Richards J, Russo JE, et al. Evaluation of the Patient Health Questionnaire-9 Item for detecting major depression among adolescents. Pediatrics. 2010;126(6):1117-23.

12. Nourbala A, Bagheri YS, MOHAMMAD K. The validation of general health questionnaire-28 as a psychiatric screening tool. 2009.

13. Tran TD, Tran T, Fisher J. Validation of the depression anxiety stress scales (DASS) 21 as a screening instrument for depression and anxiety in a rural community-based cohort of northern Vietnamese women. BMC psychiatry. 2013;13(1):1-7.

14. Topp CW, Østergaard SD, Søndergaard S, Bech P. The WHO-5 Well-Being Index: a systematic review of the literature. Psychotherapy and psychosomatics. 2015;84(3):167-76.

15. Spitzer RL, Kroenke K, Williams JB, Löwe B. A brief measure for assessing generalized anxiety disorder: the GAD-7. Archives of internal medicine. 2006;166(10):1092-7.

16. Harrer M, Cuijpers P, Furukawa T, Ebert D. Doing meta-analysis in R: a hands-on guide. PROTECT Lab Erlangen. 2019.

17. Schwarzer G. meta: An R package for meta-analysis. R news. 2007;7(3):40-5.

18. Viechtbauer W, Viechtbauer MW. Package 'metafor'. The Comprehensive R Archive Network Package 'metafor' http://cran r-project org/web/packages/metafor/metafor pdf. 2015.

19. IntHout J, loannidis JP, Borm GF. The Hartung-Knapp-Sidik-Jonkman method for random effects meta-analysis is straightforward and considerably outperforms the standard DerSimonian-Laird method. BMC medical research methodology. 2014;14(1):1-12. 
20. Higgins JP, Thompson SG, Deeks JJ, Altman DG. Measuring inconsistency in meta-analyses. Bmj. 2003;327(7414):557-60.

21. Olkin I, Dahabreh IJ, Trikalinos TA. GOSH-a graphical display of study heterogeneity. Research synthesis methods. 2012;3(3):214-23.

22. Duval S, Tweedie R. Trim and fill: a simple funnel-plot-based method of testing and adjusting for publication bias in meta-analysis. Biometrics. 2000;56(2):455-63.

23. Kim SY, Park JE, Lee YJ, Seo H-J, Sheen S-S, Hahn S, et al. Testing a tool for assessing the risk of bias for nonrandomized studies showed moderate reliability and promising validity. Journal of clinical epidemiology. 2013;66(4):408-14.

24. Keles B, McCrae N, Grealish A. A systematic review: the influence of social media on depression, anxiety and psychological distress in adolescents. International Journal of Adolescence and Youth. 2020;25(1):79-93.

25. McCrae N, Gettings S, Purssell E. Social media and depressive symptoms in childhood and adolescence: A systematic review. Adolescent Research Review. 2017;2(4):315-30.

26. Marino C, Gini G, Vieno A, Spada MM. The associations between problematic Facebook use, psychological distress and well-being among adolescents and young adults: A systematic review and meta-analysis. Journal of Affective Disorders. 2018;226:274-81.

27. Saha S, Scott J, Varghese D, McGrath J. Anxiety and depressive disorders are associated with delusional-like experiences: a replication study based on a National Survey of Mental Health and Wellbeing. BMJ open. 2012;2(3):e001001.

28. Faden J, Levin J, Mistry R, Wang J. Delusional disorder, erotomanic type, exacerbated by social media use. Case reports in psychiatry. 2017;2017.

29. Person B, Sy F, Holton K, Govert B, Liang A. Fear and stigma: the epidemic within the SARS outbreak. Emerging infectious diseases. 2004;10(2):358.

30. Soraci P, Ferrari A, Abbiati FA, Del Fante E, De Pace R, Urso A, et al. Validation and psychometric evaluation of the Italian version of the Fear of COVID-19 Scale. International Journal of Mental Health and Addiction. 2020:1-10.

31. Morahan-Martin J, Schumacher P. Loneliness and social uses of the Internet. Computers in human behavior. 2003;19(6):659-71.

\section{Figures}

\section{Figure 1}

Flowchart of literature search and selection of the publications 
Figure 2

Forest plot for social media exposure and symptoms of mental health (i.e. anxiety \& depression) in crosssectional studies. Estimates presented in odds ratios (OR).

\section{Supplementary Files}

This is a list of supplementary files associated with this preprint. Click to download.

- YoungrongSupples.pdf 